\title{
An Algorithm to Determine Driver's Fatigue Driving According to Characteristic Parameters Based on the Theory of Rough Set Importance
}

\author{
Hongxu Chen ${ }^{\mathrm{a})}$, Yifeng Xue*, b), Chen $\mathrm{Xu}^{\mathrm{c}}$, Xiaofan Wang, Longtao Pei and \\ Yanhui Han
}

School of Jilin University, Changchun 130000, China.

a) $913898217 @ q q . c o m$
b)120767727@qq.com
c)1098489671@qq.com

\begin{abstract}
The driver's fatigue driving accounts for lots of traffic accidents. Through the study of the driver's behavior characteristics,we collect the steering wheel angle, angular velocity in real time, grip force and pluse through indoor simulation experiments.After calculation, we can get these parameters of the data of zero-speed percentage, average absolute value of rotation angle, standard deviation of rotation angle, angle entropy,driver's grip force and driver's pulse.Based on the theroy of rough set importance,we calculate the weight of each signal and obtain the correlation between driving behavior signals and fatigue driving.
\end{abstract}

Keywords: Fatigue Driving, Parameters, Rough Set Importance, Traffic Accidents

\section{INTRODUCTION}

With the development of industry and economy, thousands of millions of families have their own automobiles, but at the same time, traffic accidents has also caused tragedies for countless families. In China, with the popularization of private cars, there are increasingly traffic accidents caused by fatigue driving, and more than $20 \%$ of traffic accidents are directly caused by fatigue driving. However, in fact, traffic accidents caused by driving fatigue are difficult to define, so the proportion of accidents caused by fatigue is much higher than $20 \%[1,2]$.

Talking about the fatigue driving detection based on the driver's behaviors on driving, there are many people doing relative researches. Thum Chia Chieh gave a warning to the driver's fatigue through analysing of driver's hand-held steering wheel pressure change [3]. Baulk found that the driver's driving ability, especially the lateral position of the vehicle, has a good correlation with the reaction time and the reaction time can be used as an objective evaluation criterion for driving fatigue [4]. In China, Qingning Niu collected the steering wheel angle signals during the driving through simulation experiments, and established a fatigue driving judgment model based on the steering wheel angle through the four-rotation angle feature extracted by the support vector machine method [5].

In rough set theory, the importance of attributes reflects the degree of change before and after removing one or some attributes. If the corresponding classification change, after removing this attribute, is relatively large, the importance of the attribute is great. Otherwise the importance is small [6].

In this experiments, we collect the signals of the steering wheel angle, angular velocity in real time, grip force and pluse, after calculation, get six parameters of the data of zero-speed percentage, average absolute value of rotation angle, standard deviation of rotation angle, angle entropy, driver's grip force and driver's pulse. The weights of the extracted signals are calculated according to the theory of rough set importance. The weights reflect the correlation between each of parameter and driver fatigue, which provides support for further search for the fatigue warning according to the driver behaviors. 


\section{EXPERIMENTAL DEVICE}

In the experiment, drivers will drive Logitech G27 steering wheel pedal set to simulate real driving. Steering wheel angle and angular velocity are acquired through accelerometer. Through RFP602 film pressure sensor, we acquire the signal of steering wheel grip and through YK1303P heart rate sensor we could acquire the signal of driver's pulse.

Experimental device: Logitech G27 steering wheel pedal set, Freescale chip (MC9S12XS128MAA), E6B2CWZ3E photoelectric encoder, RFP602 film pressure sensor, YK1303P heart rate sensor.

\section{ROUGH SET IMPORTANCE}

\subsection{Rough set independence}

For all $\mathrm{B}, \mathrm{E} \subseteq \mathrm{A}, \mathrm{B}$ depends in degree $\mathrm{k}(0 \leq \mathrm{k} \leq 1)$ on $\mathrm{E}$, which is $\mathrm{E} \Rightarrow \mathrm{kB}$, where The expression POSE(B) is called the positive region of the partition $\mathrm{U} / \mathrm{B}$ with respect to $\mathrm{E}$, and $\|$.$\| is the cardinality of a set [7].$

$$
\mathrm{k}=r_{E}(B)=\frac{\left\|P O S_{E}(B)\right\|}{\|U\|}
$$

\subsection{Rough set importance}

In dataset $\mathrm{S}=<\mathrm{U}, \mathrm{A}=\mathrm{C} \cup \mathrm{d}, \mathrm{V}, \mathrm{f}>, \forall \mathrm{B} \subseteq \mathrm{C}$, the ID of $\mathrm{B}$, which is related to the decision attribute $\mathrm{d}$ (in short, the related ID of B), is denoted by $R I_{C}^{d}(B)$ and defined as

$$
R I_{C}^{d}(B)=r_{C}(d)-r_{C \backslash B}(d)
$$

When $\mathrm{B}=\{\mathrm{a}\}, R I_{C}^{d}(a)$ is the related ID of a [7].

\section{ALGORITHM DESIGN}

The algorithm is implemented by MATLAB software, the aim of which is to calculate the weight and sort of six parameters, that is, the data of zero-speed percentage, average absolute value of rotation angle, standard deviation of rotation angle, angle entropy, driver's grip force and driver's pulse.

Algorithmic process is shown as follow, record the above 6 parameters as attributes A, B, C, D, E, F.

Step 1: Take decision attribute as set $\mathrm{D}$ and condition attribute as set $\mathrm{C}$.

Step 2: Classification of decision attributes, remembering 0 for lucidness and 1 for fatigue. Constructing an array of cells, which is $\mathrm{DE}=$ cell $(1,2)$.

Step 3: Classification of conditional attributes and construction of 6-dimensional array of cells as DAE.

Step 4: Calculate $r_{C}(D)$. Selection of Matlab:

B1= ismember (DAE $\{\mathrm{a} 1+1, \mathrm{a} 2+1, \mathrm{a} 3+1, \mathrm{a} 4+1, \mathrm{a} 5+1, \mathrm{a} 6+1\}$,DE $\{\mathrm{a} 7\})$;

// Determine whether the cell in DAE appears in DE.

$\mathrm{B} 2=\operatorname{all}(\mathrm{B} 1)$;

$\mathrm{B} 3=\operatorname{any}(\mathrm{B} 1)$;

if $\mathrm{B} 2 \& \& \mathrm{~B} 3$

[aa,bb]=size(DAE $\{\mathrm{a} 1+1, \mathrm{a} 2+1, \mathrm{a} 3+1, \mathrm{a} 4+1, \mathrm{a} 5+1, \mathrm{a} 6+1, \mathrm{a} 7+1\})$;

// Calculate the number of cellular elements in the DAE appearing in the DE.

$[\mathrm{m}, \mathrm{n}]=\operatorname{size}(\mathrm{C})$;

$r_{C}(D)=\mathrm{aa} / \mathrm{m}$;

Step 5: Remove attribute A and calculate $r_{C \backslash A}(D)$.

Selection of Matlab:

C10=ismember(DAE $\{1, \mathrm{a} 2+1, \mathrm{a} 3+1, \mathrm{a} 4+1, \mathrm{a} 5+1, \mathrm{a} 6+1\}, \mathrm{DE}\{\mathrm{a} 7\})$; 


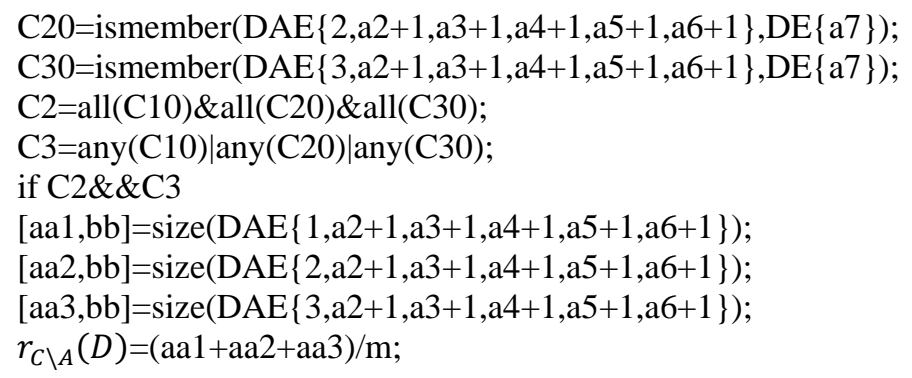

Step 6-10: Successively remove attribute B, C, D, E, F and calculate $r_{C \backslash B}(D), r_{C \backslash C}(D), r_{C \backslash D}(D), r_{C \backslash E}(D)$, $r_{C \backslash F}(D)$. Repeat step 5.

Step 11: Successively output the weight of every attribute. For example, the weight of the data of zero-speed percentage is $r_{C}(D)-r_{C \backslash A}(D)$.

\section{CONCLUSION}

After the software of Matlab running, the weights (importance) of each parameter to determine whether the driver is in a stage of fatigue are shown in Figure 1.

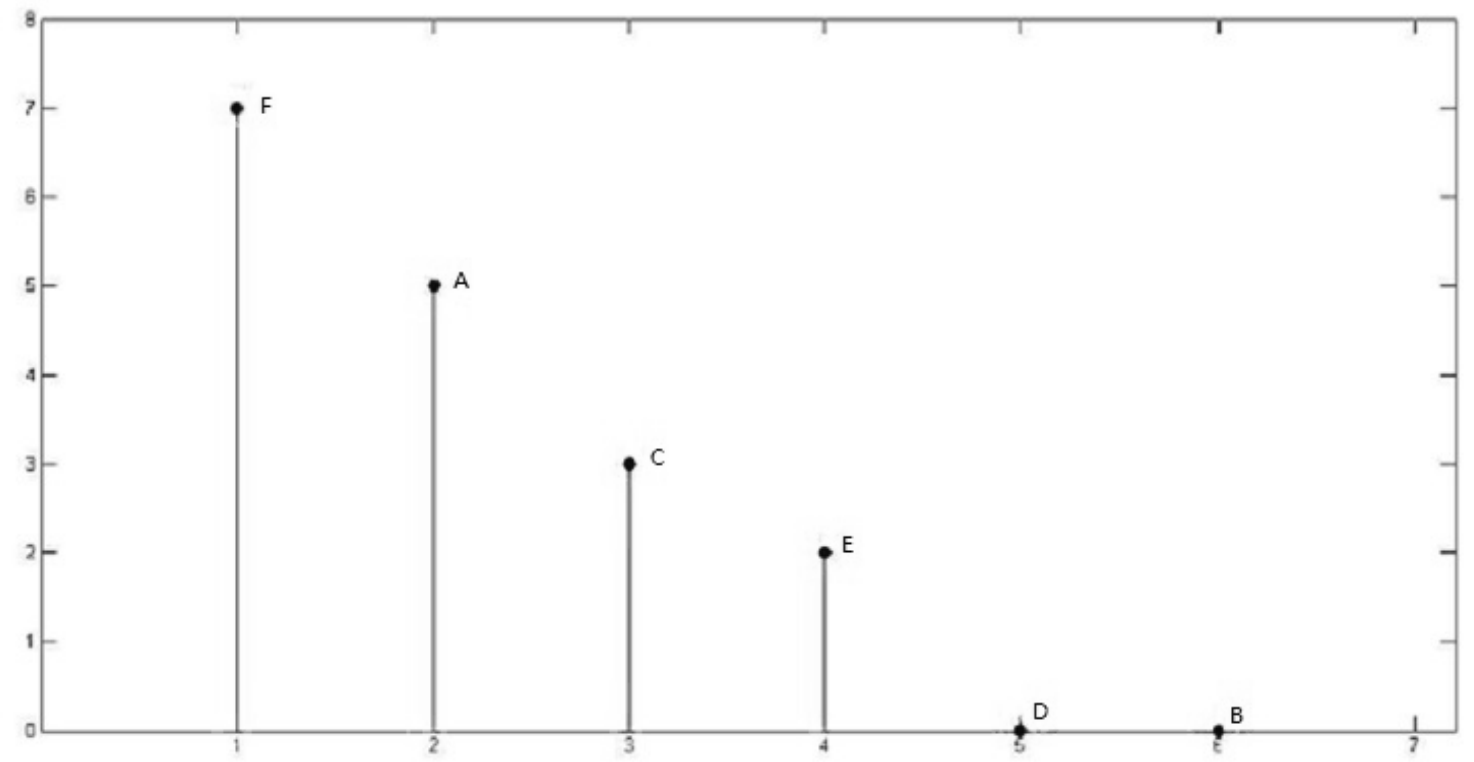

Figure 1. The weights (importance) of each parameter to determine whether the driver is in a stage of fatigue are

Through analysing, we can get a conclusion that, the pulse parameter $(\mathrm{F})$ has the largest weight for determining driver fatigue, that is, the parameter of pulse has the largest bearing on the fatigue driving. The order of the rest of the parameters' weight are successively the data of zero-speed percentage (A), standard deviation of rotation angle (C) , driver's grip force (E). Angle entropy (D) and average absolute value of rotation angle (B) have little effect when determining whether the driver is fatigued.

\section{REFERENCES}

1. Wu Weiquan.Hazard and Prevention of Fatigue Driving[J].Science \& Technology Outlook,2015(35).

2. Wu Chunying.Discussion on Research Progress of Automobile Driver Fatigue Monitoring Technology[J].Mechatronics,2007,13 (4): 43-47. 
3. Thum Chia chieh Mustafa , Hussain M M , Zahedi A,et al. Driver fatigue detection using steering grip force[C]/Malaysta, IEEE:Student Conference on Research and Development, Malaysta:IEEE 2003:45-48.

4. Baulk S D, Biggs S N, Reid K J, et al.Chasing the silver bullet:measuring driver fatigue using simple and complex tasks[J]. Accident Analysis and Prevention.2008,40(1):396-402.

5. Niu Qingning.Research on fatigue driving detection method based on information fusion[D]. Changchun: Jilin University, 2014.

6. Xiao Jinsen, Sun Limin. Improved attribute significance degree based on rough set. Computer Engineering and applications[J], 2017, 53 (3) : 174-176.

7. Dan Hu, Xianchuan Yu. Statistical Inference of Rough Set Dependence and Importance Analysis. IEEE Transactions on Fuzzy Systems[J], 2013, Vol.21: 1070-1079. 